Article

\title{
Dust-Ion-Acoustic Rogue Waves in a Dusty Plasma Having Super-Thermal Electrons
}

\author{
Akib Al Noman ${ }^{1}$, Md Khairul Islam ${ }^{1, *}$, Mehedi Hassan ${ }^{1}$, Subrata Banik ${ }^{1,2}{ }^{\oplus}$, Nure Alam Chowdhury ${ }^{3} \mathbb{D}^{\text {, }}$ \\ Abdul Mannan ${ }^{1}$ (D) and A. A. Mamun ${ }^{1}$ (D) \\ 1 Department of Physics, Jahangirnagar University, Dhaka 1342, Bangladesh; \\ noman179physics@gmail.com (A.A.N.); hassan206phy@gmail.com (M.H.); bsubrata.37@gmail.com) (S.B.); \\ abdulmannan@juniv.edu (A.M.); mamun_phys@juniv.edu (A.A.M.) \\ 2 Atomic Energy Centre, Health Physics Division, Dhaka 1000, Bangladesh \\ 3 Atomic Energy Centre, Plasma Physics Division, Dhaka 1000, Bangladesh; nurealam1743phy@gmail.com \\ * Correspondence: islam.stu2018@juniv.edu
}

check for updates

Citation: Noman, A.A.; Islam, M.K.; Hassan, M.; Banik, S.; Chowdhury, N.A.; Mannan, A.; Mamun, A.A. Dust-Ion-Acoustic Rogue Waves in a Dusty Plasma Having Super-Thermal Electrons. Gases 2021, 1, 106-116. https://doi.org/10.3390/gases1020009

Academic Editor: Ben J. Anthony

Received: 22 April 2021

Accepted: 03 June 2021

Published: 11 June 2021

Publisher's Note: MDPI stays neutral with regard to jurisdictional claims in published maps and institutional affiliations.

Copyright: (c) 2021 by the authors. Licensee MDPI, Basel, Switzerland. This article is an open access article distributed under the terms and conditions of the Creative Commons Attribution (CC BY) license (https:// creativecommons.org/licenses/by/ $4.0 /)$.

\begin{abstract}
The standard nonlinear Schrödinger Equation (NLSE) is one of the elegant equations to find detailed information about the modulational instability criteria of dust-ion-acoustic (DIA) waves and associated DIA rogue waves (DIARWs) in a three-component dusty plasma medium with inertialess super-thermal kappa distributed electrons, and inertial warm positive ions and negative dust grains. It can be seen that the plasma system supports both fast and slow DIA modes under consideration of inertial warm ions along with inertial negatively charged dust grains. It is also found that the modulationally stable parametric regime decreases with $\kappa$. The numerical analysis has also shown that the amplitude of the first and second-order DIARWs decreases with ion temperature. These results are to be considered the cornerstone for explaining the real puzzles in space and laboratory dusty plasmas.
\end{abstract}

Keywords: plasma; laboratory dusty plasmas; NLSE; DPM; DIAWs

\section{Introduction}

The size, mass, charge, and ubiquitous existence of massive dust grains in both space (viz., cometary tails [1-3], planetary rings [1], Earth's ionospheres [1], magnetosphere [2], ionosphere [2], aerosols in the astrosphere [3-5], nebula, and interstellar medium [4], etc.) and laboratory (viz., ac-discharge, plasma crystal [4], rf-discharges [4], Q-machine, and nano-materials [6], etc.) plasmas do not only change the dynamics of the dusty plasma medium (DPM), but also change the mechanism to the formation of various nonlinear electrostatic excitations [7-9], viz., dust-acoustic (DA) solitary waves (DASWs) [2], DA shock waves (DASHWs) [10], dust-ion-acoustic (DIA) solitary waves (DIASWs) [2], and DIA rogue waves (DIARWs) [11-13], etc.

The activation of the long range gravitational and Coulomb force fields is the main cause to generate non-equilibrium species as well as the high energy tail in space environments, viz., terrestrial plasma-sheet [14], magneto-sheet, auroral zones [14], mesosphere, radiation belts [15], magnetosphere, and ionosphere [15], etc. The Maxwellian velocity distribution function, in which the dynamics of the non-equilibrium species is not considered, is not enough to describe the intrinsic mechanism of the high energy tail in space environments [16]. The $\kappa$-distribution function, in which the dynamics of the non-equilibrium species is also considered, is suitable for explaining the high energy tail in space environments [16]. The non-equilibrium properties of these species are recognized by the magnitude of $\kappa$ in super-thermal $\kappa$-distribution [16]. The $\kappa$-distribution is normalizable for a range of values of $\kappa$ from $\kappa>3 / 2$ to $\kappa \rightarrow \infty$, and the non-equilibrium properties of these species are considerable when the value of $\kappa$ tends to $3 / 2$ [1]. Eslami et al. [1] observed that the velocity of the DIASWs increases with decreasing the value of $\kappa$ in a DPM. 
Shahmansouri and Tribeche [2] considered an electron depleted DPM with super-thermal plasma species to investigate DASWs, and reported that the amplitude of the DASWs increases while the width of the DASWs decreases with the decreasing value of $\kappa$, which means that the super-thermality of the plasma species leads to narrower and spiky solitons. Ferdousi et al. [10] numerically observed that the height of the positive DASHWs increases while negative DASHWs decreases with increasing the value of $\kappa$ in a multi-component DPM in the presence of super-thermal electrons.

The nonlinear and dispersive properties of plasma medium are the prime reasons to determine the modulational instability (MI) criteria of various kinds of waves in the presence of external perturbation, and are governed by the standard nonlinear Schrödinger Equation (NLSE), which can be derived by employing reductive perturbation method (RPM) [17-22]. The rational solution of the NLSE is also known as freak waves, giant waves, or rogue waves (RWs), in which a large amount of energy can concentrate into a small area, and the height of the RWs is almost three times greater then the height of associated normal carrier waves. Initially, RWs are identified only in the ocean and are considered as a destructive sign of nature, which can sink the ship or destroy the houses in the bank of the ocean. Nowadays, RWs can also be observed in optics, stock market, biology, plasma physics, etc. Gill et al. [7] investigated the MI of the DAWs in a multi-component DPM and found that the critical wave number $\left(k_{c}\right)$, which defines the modulationally stable and unstable parametric regimes, decreases with the increase in the value of $\kappa$. Amin et al. [17] studied the propagation of nonlinear electrostatic DAWs and DIAWs, and their MIs in a three-component DPM. Jukui and He [18] demonstrated the amplitude modulation of spherical and cylindrical DIAWs. Saini and Kourakis [19] considered a three-component DPM with inertial highly charged massive dust grains and inertialess electrons and ions to study the MI of DAWs, and highlighted that the angular frequency of the DAWs increases with the super-thermality of the plasma species, and the stable parametric regime decreases with an increase in the value of negative dust number density.

In DAWs [23-27], the moment of inertia is provided by the mass of the dust grains, and restoring force is provided by the thermal pressure of the ions and electrons. On the other hand, in DIAWs [28-32], the moment of inertia is provided by the mass of the ions, and restoring force is provided by the thermal pressure of the electrons in the presence of immobile dust grains. The mass and charge of the dust grains are considerably larger than those of the ions, while the mass and charge of the ion are considerably larger than those of the electron. It may be noted here that in the DIAWs, if anyone considers the thermal effects of the ions, then it is important to consider the moment of inertia of the ions along with the dust grains in the presence of inertialess electrons [33-35]. This means that the consideration of the thermal effects of the ions highly contributes to the moment of inertia along with inertial dust grains to generate DIAWs in a DPM having inertialess electrons [33-35]. In the present work, we are interested to investigate the nonlinear propagation of DIA waves and associated DIARWs in which the moment of inertia is provided by the mass of the inertial warm ions and negatively charged dust grains, and the restoring force is provided by the thermal pressure of the inertialess electrons in a three-component DPM (dust-ion-electrons) by using the standard NLSE.

The outline of the paper is as follows: The governing equations describing our plasma model are presented in Section 2. The derivation of NLSE is demonstrated in Section 3. The Modulational instability and rogue waves are given in Section 4. Results and discussion are given in Section 5. The conclusion is provided in Section 6.

\section{Governing Equations}

We consider an unmagnetized, fully ionized, and collisionless three-component DPM comprising super-thermal electrons, positively charged inertial warm ions, and negatively charged dust grains. At equilibrium, the overall charge neutrality condition of our plasma system can be written as $n_{e 0}+Z_{d} n_{d 0}=Z_{i} n_{i 0}$, where $n_{e 0}, n_{d 0}$, and $n_{i 0}$ are the equilibrium 
electron, dust, and ion number densities, respectively, and $Z_{d}\left(Z_{i}\right)$ is the charge state of the negative (positive) dust grain (ion). The normalized equations describing the system can be written as

$$
\begin{aligned}
& \frac{\partial n_{d}}{\partial t}+\frac{\partial}{\partial x}\left(n_{d} u_{d}\right)=0 \\
& \frac{\partial u_{d}}{\partial t}+u_{d} \frac{\partial u_{d}}{\partial x}=\mu_{1} \frac{\partial \phi}{\partial x} \\
& \frac{\partial n_{i}}{\partial t}+\frac{\partial}{\partial x}\left(n_{i} u_{i}\right)=0, \\
& \frac{\partial u_{i}}{\partial t}+u_{i} \frac{\partial u_{i}}{\partial x}+\mu_{2} n_{i} \frac{\partial n_{i}}{\partial x}=-\frac{\partial \phi}{\partial x}, \\
& \frac{\partial^{2} \phi}{\partial x^{2}}+n_{i}=\left(1-\mu_{3}\right) n_{e}+\mu_{3} n_{d}
\end{aligned}
$$

where $n_{d}\left(n_{i}\right)$ is the dust (ion) number density normalized by the equilibrium value $n_{d 0}\left(n_{i 0}\right)$; $u_{d}\left(u_{i}\right)$ is the dust (ion) fluid speed normalized by the ion sound speed $C_{i}=\left(Z_{i} k_{B} T_{e} / m_{i}\right)^{1 / 2}$ (where $T_{e}$ being the super-thermal electron temperature, $m_{i}$ is the ion rest mass, and $k_{B}$ is the Boltzmann constant); $\phi$ is the electrostatic wave potential normalized by $k_{B} T_{e} / e$ (with $e$ being the electron charge). The time variable $t$ is normalized by $\omega_{P_{i}}^{-1}=\left[m_{i} /\left(4 \pi e^{2} Z_{i}^{2} n_{i 0}\right)\right]^{1 / 2}$ and the space variable $x$ is normalized by $\lambda_{D_{i}}=\left(k_{B} T_{e} / 4 \pi e^{2} Z_{i} n_{i 0}\right)^{1 / 2}$. The pressure of the ion is expressed as $P_{i}=P_{i 0}\left(N_{i} / N_{i 0}\right)^{\gamma}$, with $P_{i 0}=n_{i 0} k_{B} T_{i}$ being the equilibrium pressure of the ion, $T_{i}$ being the warm ion temperature, and $\gamma=(N+2) / N$ (where $N$ be the degree of freedom and for one dimensional case $N=1$, then $\gamma=3$ ). Other plasma parameters are defined as $\mu_{1}=\rho \mu, \rho=Z_{d} / Z_{i}, \mu=m_{i} / m_{d}, \mu_{2}=3 T_{i} / Z_{i} T_{e}$, and $\mu_{3}=Z_{d} n_{d 0} / Z_{i} n_{i 0}$. The expression for the number density of the super-thermal electrons (following the $\kappa$ distribution) can be expressed as [36]

$$
n_{e}=\left[1-\frac{\phi}{\kappa-3 / 2}\right]^{-\kappa+\frac{1}{2}}=1+n_{1} \phi+n_{2} \phi^{2}+n_{3} \phi^{3}+\cdots,
$$

where $n_{1}=(2 \kappa-1) /(2 \kappa-3), n_{2}=[(2 \kappa-1)(2 \kappa+1)] / 2(2 \kappa-3)^{2}$, and $n_{3}=[(2 \kappa-$ $1)(2 \kappa+1)(2 \kappa+3)] / 6(2 \kappa-3)^{3}$. The parameter $\kappa$ generally stands for super-thermality, which measures the deviation of the plasma particles from Maxwellian distribution. Now, by substituting Equation (6) into Equation (5) and expanding up to third order in $\phi$, we get

$$
\frac{\partial^{2} \phi}{\partial x^{2}}+n_{i}+\mu_{3}=1+\mu_{3} n_{d}+A_{1} \phi+A_{2} \phi^{2}+A_{3} \phi^{3}+\cdots,
$$

where $A_{1}=n_{1}\left(1-\mu_{3}\right), A_{2}=n_{2}\left(1-\mu_{3}\right)$, and $A_{3}=n_{3}\left(1-\mu_{3}\right)$.

\section{Derivation of the NLSE}

To study the MI of the DIAWs, we want to derive the NLSE by employing the RPM. First, we can write the stretched co-ordinates in the following form [37-39]

$$
\begin{aligned}
& \xi=\epsilon\left(x-v_{g} t\right), \\
& \tau=\epsilon^{2} t
\end{aligned}
$$

where $v_{g}$ is the group velocity and $\epsilon(0<\epsilon<1)$ is a small parameter. We can write the dependent variables $\left(n_{d}, u_{d}, n_{i}, u_{i}\right.$, and $\left.\phi\right)$ as [37-39] 


$$
\begin{aligned}
& n_{d}=1+\sum_{m=1}^{\infty} \epsilon^{m} \sum_{l=-\infty}^{\infty} n_{d l}^{(m)}(\xi, \tau) \mathrm{e}^{i l(k x-\omega t),} \\
& u_{d}=\sum_{m=1}^{\infty} \epsilon^{m} \sum_{l=-\infty}^{\infty} u_{d l}^{(m)}(\xi, \tau) \mathrm{e}^{i l(k x-\omega t)}, \\
& n_{i}=1+\sum_{m=1}^{\infty} \epsilon^{m} \sum_{l=-\infty}^{\infty} n_{i l}^{(m)}(\xi, \tau) \mathrm{e}^{i l(k x-\omega t),} \\
& u_{i}=\sum_{m=1}^{\infty} \epsilon^{m} \sum_{l=-\infty}^{\infty} u_{i l}^{(m)}(\xi, \tau) \mathrm{e}^{i l(k x-\omega t)}, \\
& \phi=\sum_{m=1}^{\infty} \epsilon^{m} \sum_{l=-\infty}^{\infty} \phi_{l}^{(m)}(\xi, \tau) \mathrm{e}^{i l(k x-\omega t)},
\end{aligned}
$$

where $k$ and $\omega$ are the real variables representing the carrier wave number and frequency, respectively. The derivative operators can be written as [37-39]

$$
\begin{aligned}
\frac{\partial}{\partial x} & \rightarrow \frac{\partial}{\partial x}+\epsilon \frac{\partial}{\partial \xi^{\prime}} \\
\frac{\partial}{\partial t} & \rightarrow \frac{\partial}{\partial t}-\epsilon v_{g} \frac{\partial}{\partial \xi}+\epsilon^{2} \frac{\partial}{\partial \tau} .
\end{aligned}
$$

Now, by substituting Equations (8)-(16) into Equations (1)-(4) and (7), and collecting the terms containing $\epsilon$, the first order $(m=1$ with $l=1)$ reduced equations can be written as

$$
\begin{aligned}
u_{d 1}^{(1)} & =-\frac{k \mu_{1}}{\omega} \phi_{1}^{(1)}, & n_{d 1}^{(1)} & =-\frac{\mu_{1} k^{2}}{\omega^{2}} \phi_{1}^{(1)}, \\
u_{i 1}^{(1)} & =-\frac{k \omega}{\mu_{2} k^{2}-\omega^{2}} \phi_{1}^{(1)}, & n_{i 1}^{(1)} & =-\frac{k^{2}}{\mu_{2} k^{2}-\omega^{2}} \phi_{1}^{(1)},
\end{aligned}
$$

these relations provide the dispersion relations of DIAWs. Now, the dispersion relations of DIAWs are

$$
\omega^{2} \equiv \omega_{f}^{2}=\frac{k^{2} M+k^{2} \sqrt{M^{2}-4 G H}}{2 G},
$$

and

$$
\omega^{2} \equiv \omega_{s}^{2}=\frac{k^{2} M-k^{2} \sqrt{M^{2}-4 G H}}{2 G},
$$

where $M=1+\mu_{1} \mu_{3}+\mu_{2} k^{2}+A_{1} \mu_{2}, G=A_{1}+k^{2}$, and $H=\mu_{1} \mu_{2} \mu_{3}$. In Equation (19) and (20), to get the positive value of $\omega$, the condition $M^{2}>4 G H$ must be satisfied. In the fast $\left(\omega_{f}\right)$ DIA mode, both inertial ion and dust oscillate in phase with the inertialess electrons. While in the slow $\left(\omega_{s}\right)$ DIA mode, one of the inertial elements ion (dust) oscillates in phase with the inertialess electrons, and other inertial element dust (ion) oscillates in anti-phase with them $[40,41]$. Both the fast $\left(\omega_{f}\right)$ and slow $\left(\omega_{s}\right)$ DIA modes have been analyzed numerically in Figure 1 in the presence of super-thermal electrons. Figure 1 (left panel) shows that the frequency of the DIAWs can be higher than ion-plasma frequency. In this regard, we note that in the absence of dust, the frequency of the ion-acoustic waves is always less than the ion-plasma or ion-Langmuir frequency. However, the phase speed of the DIAWs increases with the magnitude of the dust charge $\left(Z_{d}\right)$ and dust number density $\left(n_{d 0}\right)$. This is due to the extra space charge electric field created by the highly negative charged dust grains. This is theoretically predicted by Shukla and Silin [42], and experimentally observed by Barkan et al. [43]. Thus, as the magnitude of the dust charge $\left(Z_{d}\right)$ or dust number density $\left(n_{d 0}\right)$ increases, the frequency of the DIAWs increases; even it can exceed the ion-plasma or ion-Langmuir frequency. On the other hand, the dispersion 
curve of the slow DIA mode shown in Figure 1 (right panel) clearly indicates that the frequency of the slow DIA mode is always less than the ion-plasma or ion-Langmuir frequency even in the presence of highly negatively charged dust. The second-order (when $m=2$ with $l=1$ ) equations are given by

$$
\begin{aligned}
& u_{d 1}^{(2)}=-\frac{k \mu_{1}}{\omega} \phi_{1}^{(2)}-\frac{\mu_{1}}{i \omega} \frac{\partial \phi_{1}^{(1)}}{\partial \xi}+\frac{k v_{g} \mu_{1}}{i \omega^{2}} \frac{\partial \phi_{1}^{(1)}}{\partial \xi}, \\
& n_{d 1}^{(2)}=-\frac{\mu_{1} k^{2}}{\omega^{2}} \phi_{1}^{(2)}+\frac{2 k \mu_{1}\left(k v_{g}-\omega\right)}{i \omega^{3}} \frac{\partial \phi_{1}^{(1)}}{\partial \xi}, \\
& u_{i 1}^{(2)}=\frac{k \omega}{\omega^{2}-\mu_{2} k^{2}} \phi_{1}^{(2)}-\frac{i\left(\omega-k v_{g}\right)\left(\omega^{2}+\mu_{2} k^{2}\right)}{\left(\omega^{2}-\mu_{2} k^{2}\right)^{2}} \frac{\partial \phi_{1}^{(1)}}{\partial \xi}, \\
& n_{i 1}^{(2)}=\frac{k^{2}}{\omega^{2}-\mu_{2} k^{2}} \phi_{1}^{(2)}-\frac{2 i k \omega\left(\omega-k v_{g}\right)}{\left(\omega^{2}-\mu_{2} k^{2}\right)^{2}} \frac{\partial \phi_{1}^{(1)}}{\partial \xi}
\end{aligned}
$$

with the compatibility condition for the group velocity of DIAW as

$$
v_{g}=\frac{2 \mu_{1} \mu_{3} k \omega\left(\omega^{2}-\mu_{2} k^{2}\right)^{2}+2 k \omega^{5}-2 k \omega^{3}\left(\omega^{2}-\mu_{2} k^{2}\right)^{2}}{2 k^{2} \mu_{1} \mu_{3}\left(\omega^{2}-\mu_{2} k^{2}\right)^{2}+2 k^{2} \omega^{4}} .
$$
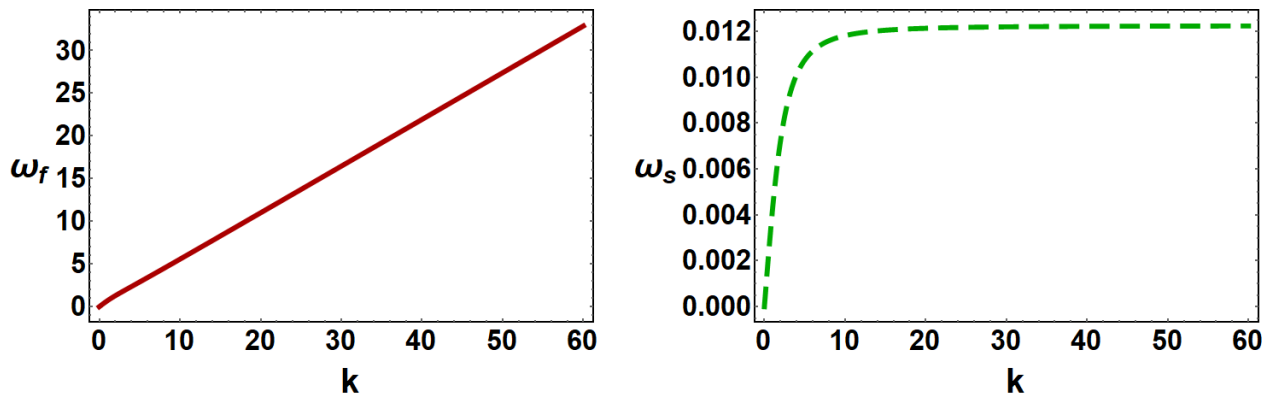

Figure 1. The variation of $\omega_{f}$ vs. $k$ (left panel) and $\omega_{s}$ vs. $k$ (right panel) when other plasma parameters are $\kappa=1.8, \rho=1 \times 10^{3}, \mu=3 \times 10^{-6}, \mu_{2}=0.3$, and $\mu_{3}=0.05$.

The coefficients of $\epsilon$ when $m=2$ with $l=2$ provides the second-order harmonic amplitudes which are found to be proportional to $\left|\phi_{1}^{(1)}\right|^{2}$

$$
\begin{gathered}
u_{d 2}^{(2)}=A_{4}\left|\phi_{1}^{(1)}\right|^{2}, \quad n_{d 2}^{(2)}=A_{5}\left|\phi_{1}^{(1)}\right|^{2}, \\
u_{i 2}^{(2)}=A_{6}\left|\phi_{1}^{(1)}\right|^{2}, \quad n_{i 2}^{(2)}=A_{7}\left|\phi_{1}^{(1)}\right|^{2}, \quad \phi_{2}^{(2)}=A_{8}\left|\phi_{1}^{(1)}\right|^{2},
\end{gathered}
$$

where

$$
\begin{aligned}
& A_{4}=\frac{k^{3} \mu_{1}^{2}-2 A_{8} \mu_{1} k \omega^{2}}{2 \omega^{3}}, \quad A_{5}=\frac{3 k^{4} \mu_{1}^{2}-2 A_{8} \mu_{1} k^{2} \omega^{2}}{2 \omega^{4}}, \\
& A_{6}=-\frac{2 A_{8} k \omega\left(\mu_{2} k^{2}-\omega^{2}\right)^{2}+3 \mu_{2} \omega k^{5}+k^{3} \omega^{3}}{2\left(\mu_{2} k^{2}-\omega^{2}\right)^{3}}, \\
& A_{7}=-\frac{2 A_{8} k^{2}\left(\mu_{2} k^{2}-\omega^{2}\right)^{2}+3 \omega^{2} k^{4}+\mu_{2} k^{6}}{2\left(\mu_{2} k^{2}-\omega^{2}\right)^{3}}, \\
& A_{8}=\frac{\left(2 A_{2} \omega^{4}+3 \mu_{1}^{2} \mu_{3} k^{4}\right)+3 \omega^{6} k^{4}+\mu_{2} k^{6} \omega^{4}}{2 \omega^{2}\left[\left(k^{2} \mu_{1} \mu_{3}-4 k^{2} \omega^{2}-A_{1} \omega^{2}\right)-k^{2} \omega^{2}\left(\mu_{2} k^{2}-\omega^{2}\right)^{-1}\right]} .
\end{aligned}
$$

When $m=3$ with $l=0$ and $m=2$ with $l=0$ lead to zeroth harmonic modes as follows 


$$
\begin{gathered}
u_{d 0}^{(2)}=A_{9}\left|\phi_{1}^{(1)}\right|^{2}, \quad n_{d 0}^{(2)}=A_{10}\left|\phi_{1}^{(1)}\right|^{2}, \\
u_{i 0}^{(2)}=A_{11}\left|\phi_{1}^{(1)}\right|^{2}, \quad n_{i 0}^{(2)}=A_{12}\left|\phi_{1}^{(1)}\right|^{2}, \quad \phi_{0}^{(2)}=A_{13}\left|\phi_{1}^{(1)}\right|^{2},
\end{gathered}
$$

where

$$
\begin{aligned}
& A_{9}=\frac{k^{2} \mu_{1}^{2}-\mu_{1} \omega^{2} A_{13}}{\omega^{2} v_{g}}, \quad A_{10}=\frac{2 k^{3} \mu_{1}^{2} v_{g}+k^{2} \mu_{1}^{2} \omega-\mu_{1} \omega^{3} A_{13}}{v_{g}^{2} \omega^{3}}, \\
& A_{11}=\frac{v_{g} A_{13}\left(\omega^{2}-\mu_{2} k^{2}\right)^{2}+2 \mu_{2} \omega k^{3}+\mu_{2} k^{4} v_{g}+k^{2} \omega^{2} v_{g}}{\left(\omega^{2}-\mu_{2} k^{2}\right)^{2}\left(v_{g}^{2}-\mu_{2}\right)}, \\
& A_{12}=\frac{A_{13}\left(\omega^{2}-\mu_{2} k^{2}\right)^{2}+2 \omega k^{3} v_{g}+\mu_{2} k^{4}+k^{2} \omega^{2}}{\left(\omega^{2}-\mu_{2} k^{2}\right)^{2}\left(v_{g}^{2}-\mu_{2}\right)}, \\
& A_{13}=\frac{\left(\omega^{2}-\mu_{2} k^{2}\right)^{2} \times F_{1}-v_{g}^{2} \omega^{3}\left(2 \omega k^{3} v_{g}+\mu_{2} k^{4}+k^{2} \omega^{2}\right)}{\omega^{3}\left(\omega^{2}-\mu_{2} k^{2}\right)^{2}\left[\mu_{1} \mu_{3}\left(v_{g}^{2}-\mu_{2}\right)+v_{g}^{2}-A_{1} v_{g}^{2}\left(v_{g}^{2}-\mu_{2}\right)\right]},
\end{aligned}
$$

where $F_{1}=\left(v_{g}^{2}-\mu_{2}\right)\left(2 A_{2} v_{g}^{2} \omega^{3}+2 k^{3} \mu_{1}^{2} \mu_{3} v_{g}+k^{2} \omega \mu_{1}^{2} \mu_{3}\right)$. Finally, the third-order harmonic modes (when $m=3$ and $l=1$ ) give a set of equations which can be reduced to the standard NLSE:

$$
i \frac{\partial \Phi}{\partial \tau}+P \frac{\partial^{2} \Phi}{\partial \tilde{\xi}^{2}}+Q|\Phi|^{2} \Phi=0
$$

where $\Phi=\phi_{1}^{(1)}$ for simplicity. In Equation (28), the dispersion coefficients $(P)$ and nonlinear coefficients $(Q)$ can be written, respectively, as

$$
\begin{aligned}
& P=-\frac{\left(\omega-k v_{g}\right)\left(\omega^{2}-\mu_{2} k^{2}\right)^{3}\left(3 k v_{g} \mu_{1} \mu_{3}-\mu_{1} \mu_{2} \omega\right)+F_{2}}{\omega\left(\omega^{2}-\mu_{2} k^{2}\right)\left[2 \mu_{1} \mu_{3} k^{2}\left(\omega^{2}-\mu_{2} k^{2}\right)^{2}+2 k^{2} \omega^{4}\right]}, \\
& Q=\frac{\omega^{3}\left(\omega^{2}-\mu_{2} k^{2}\right)^{2}\left[3 A_{3}+2 A_{2}\left(A_{8}+A_{13}\right)-F_{3}\right]}{2 \mu_{1} \mu_{3} k^{2}\left(\omega^{2}-\mu_{2} k^{2}\right)^{2}+2 k^{2} \omega^{4}},
\end{aligned}
$$

where $F_{2}=\left(2 k \omega^{6} v_{g}-2 \mu_{2} k^{2} \omega^{5}\right)\left(\omega-k v_{g}\right)+\left(\omega^{4} k v_{g}-\omega^{5}\right)\left(\omega-k v_{g}\right)\left(\omega^{2}+\mu_{2} k^{2}\right)+\omega^{4}\left(\omega^{2}-\right.$ $\left.\mu_{2} k^{2}\right)^{3}$ and $F_{3}=\left\{\left(k^{2} \omega^{2}+\mu_{2} k^{4}\right)\left(A_{7}+A_{12}\right)+2 k^{3} \omega\left(A_{6}+A_{11}\right)\right\} /\left(\omega^{2}-\mu_{2} k^{2}\right)^{2}+\left\{2 k^{3} \mu_{1} \mu_{3}\right.$ $\left.\left(A_{4}+A_{9}\right)+k^{2} \omega \mu_{1} \mu_{3}\left(A_{5}+A_{10}\right)\right\} / \omega^{3}$. It is interesting that $P$ and $Q$ of Equation (28) are the function of various plasma parameters such as carrier wave number $(k)$, ratio of ion mass to dust mass $(\mu)$, ratio of dust charge state to ion charge state $(\rho)$, and super-thermal parameter $(\kappa)$, etc.

\section{Modulational Instability and Rogue Waves}

The stable and unstable parametric regimes of the DIAWs are determined by the sign of the dispersion $(P)$ and nonlinear $(Q)$ coefficients of the standard NLSE [44-47]. The stability of DIAWs in a three-component DPM is governed by the sign of $P$ and $Q$ [44-47]. When $P$ and $Q$ are with the same sign (i.e., $P / Q>0$ ), the evolution of the DIAWs amplitude is modulationally unstable. On the other hand, when $P$ and $Q$ are with the opposite sign (i.e., $P / Q<0$ ), the DIAWs are modulationally stable in the presence of external perturbations. The plot of $P / Q$ against $k$ yields stable and unstable parametric regimes of DIAWs. The point, at which transition of $P / Q$ curve intersects with $k$-axis, is known as threshold or critical wave number $k\left(=k_{c}\right)$ [44-47]. When $P / Q>0$ and $\tilde{k}<k_{c}$, the MI growth rate $\left(\Gamma_{g}\right)$ is given by

$$
\Gamma_{g}=|P| \tilde{k}^{2} \sqrt{\frac{\tilde{k}_{c}^{2}}{\tilde{k}^{2}}-1}
$$


The first-order rational solution, which can predict the concentration of the large amount of energy in a small region in the modulationally unstable parametric regime $(P / Q>0)$ of DIAWs, of Equation (28) can be written as [48,49]

$$
\Phi_{1}(\xi, \tau)=\sqrt{\frac{2 P}{Q}}\left[\frac{4+16 i \tau P}{1+4 \xi^{2}+16 \tau^{2} P^{2}}-1\right] \exp (2 i \tau P) .
$$

and the second-order rational solution is [21]

$$
\Phi_{2}(\xi, \tau)=\sqrt{\frac{P}{Q}}\left[1+\frac{G_{2}(\xi, \tau)+i M_{2}(\xi, \tau)}{D_{2}(\xi, \tau)}\right] \exp (i \tau P),
$$

where

$$
\begin{aligned}
G_{2}(\xi, \tau)= & \frac{-\xi^{4}}{2}-6(P \xi \tau)^{2}-10(P \tau)^{4}-\frac{3 \xi^{2}}{2}-9(P \tau)^{2}+\frac{3}{8} \\
M_{2}(\xi, \tau)= & -P \tau\left[\xi^{4}+4(P \xi \tau)^{2}+4(P \tau)^{4}-3 \xi^{2}+2(P \tau)^{2}-\frac{15}{4}\right] \\
D_{2}(\xi, \tau)= & \frac{\xi^{6}}{12}+\frac{\xi^{4}(P \tau)^{2}}{2}+\xi^{2}(P \tau)^{4}+\frac{2(P \tau)^{6}}{3} \\
& +\frac{\xi^{4}}{8}+\frac{9(P \tau)^{4}}{2}-\frac{3(P \xi \tau)^{2}}{2}+\frac{9 \xi^{2}}{16}+\frac{33(P \tau)^{2}}{8}+\frac{3}{32}
\end{aligned}
$$

The nonlinear behavior of the plasma medium is considered to be responsible for the concentration of large amount of energy into tiny region.

\section{Results and Discussion}

First, we are interested to observe numerically stable and unstable parametric regimes of DIAWs in the presence of super-thermal electrons by depicting the variation of $P / Q$ with $k$ for different plasma parameters. In our present analysis, we have considered that $m_{i}=3 \times 10^{-6} m_{d}, Z_{d}=10^{3} Z_{i}$, and $T_{e}=10 T_{i}[50-53]$.

We have graphically shown the variation of $P / Q$ with $k$ in case of both fast $\left(\omega_{f}\right)$ and slow $\left(\omega_{s}\right)$ DIA modes for different values of $\kappa$ in Figure 2, and it can be seen from this figure that (a) under consideration $\omega \equiv \omega_{f}$ and $\omega \equiv \omega_{s}$, possible stable and unstable parametric regimes can be occurred for DIAWs; (b) the DIAWs become unstable for small value of $k$ (i.e., $k \simeq 1$ ) in first mode (left panel) while in slow mode (right panel) the DIAWs become unstable for large value of $k$ (i.e., $k \simeq 6$ ) for same plasma parameters; and (c) the $k_{c}$ decreases with an increase in the value of $k$, and this result agrees with the previous works [7-9].
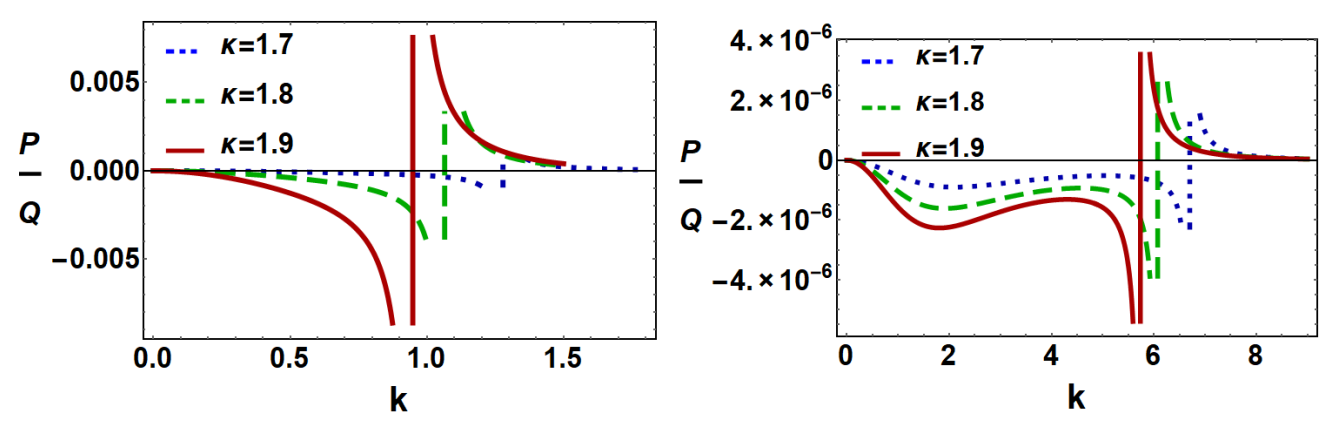

Figure 2. The variation of $P / Q$ with $k$ for different values of $\kappa$ for $\omega \equiv \omega_{f}$ (left panel) and $\omega \equiv \omega_{s}$ (right panel) when other plasma parameters are $\rho=1 \times 10^{3}, \mu=3 \times 10^{-6}, \mu_{2}=0.3$, and $\mu_{3}=0.05$.

We have numerically analyzed the variation of the MI growth rate $\left(\Gamma_{g}\right)$ of DIAWs with $\tilde{k}$ for different values of $\mu_{3}$ under consideration of fast mode shown in the left panel 
of Figure 3 by using these following plasma parameters: $k=1.3, \tilde{\Phi}_{0}=0.5, \rho=1 \times 10^{3}$, $\mu=3 \times 10^{-6}$, and $\mu_{2}=0.3$, and it is clear from this figure that the $\Gamma_{g}$, initially, increases with $\tilde{k}$, and becomes maximum for a particular value of $\tilde{k}$ (i.e., $\tilde{k} \simeq 18$ ), then decreases to zero (i.e., $\tilde{k} \simeq 25$ ). The maximum value of the $\Gamma_{g}$ increases (decreases) with the dust (ion) charge state for a fixed value of dust and ion number density (via $\mu_{3}$ ). In our present investigation, we have considered the thermal effects of ions as well as the moment of inertia of ions along with negative dust grains. So, it is important to examine how the dynamics of the plasma system changes due to the consideration of thermal effects as well as the moment of inertia of positive ions along with negative dust grains. We have numerically analyzed Equation (32) in the right panel of Figure 3 to observe the effects of ion temperature (via $\mu_{2}$ ) to the formation of first-order DIARWs. The amplitude and width of the first-order DIARWs decrease with the increase in the value of the ion temperature $\left(T_{i}\right)$ for a fixed value of ion charge state $\left(Z_{i}\right)$ and electron temperature $\left(T_{e}\right)$. The physics behind this result is that the nonlinearity as well as the amplitude of the first-order DIARWs decrease with ion temperature. So, under consideration of the thermal effect of inertial positive ion significantly changes, the dynamics of the plasma medium, and this finding agrees with the previous work [54].
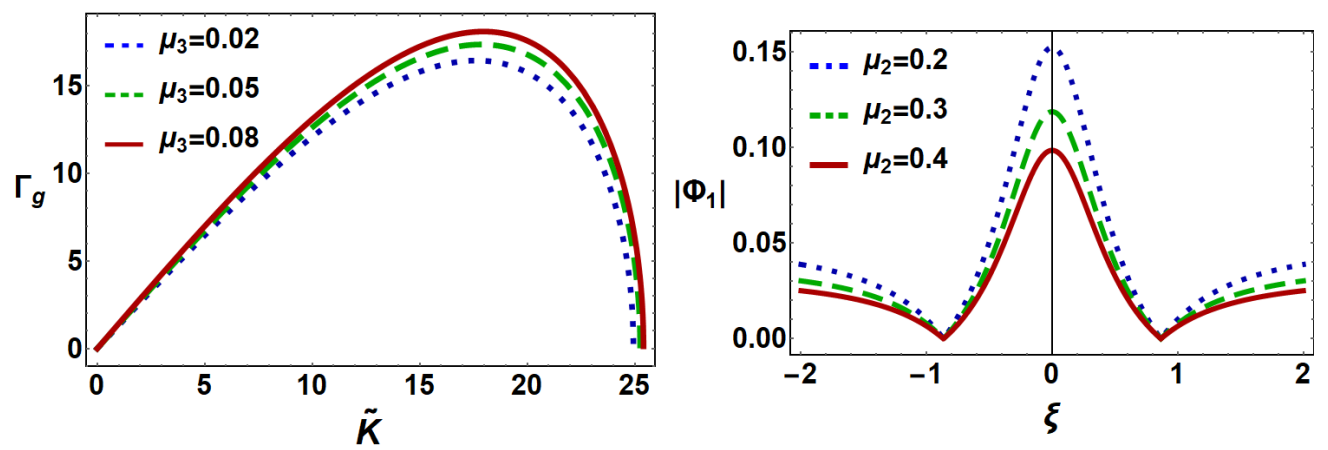

Figure 3. The variation of $\Gamma_{g}$ with $\tilde{k}$ for different values of $\mu_{3}$ (left panel) and the variation of $\left|\Phi_{1}\right|$ with $\xi$ for different values of $\mu_{2}$ (right panel) when other plasma parameters are $k=1.3, \tilde{\Phi}_{0}=0.5$, $\kappa=1.8, \rho=1 \times 10^{3}, \mu=3 \times 10^{-6}$, and $\omega \equiv \omega_{f}$.

We have presented the evaluation of the second-order DIARWs with $\xi$ for different values of $\mu_{2}$ in the left panel of Figure 4, and from this figure it is observed that (a) the second-order DIARW solutions can concentrate a large amount of energy into a small region; (b) the amplitude of the second-order rogue waves decreases (increases) with the increasing temperature of the ion (electron) for a fixed ion charge state; (b) the nonlinearity as well as the amplitude of the second-order DIARWs increases with ion charge state for a fixed value of ion and electron temperature. The right panel of Figure 4 shows a comparison between the first and second-order DIARWs and it can be seen from this figure that the amplitude of the second-order DIARWs is always higher than the first-order DIARWs for same plasma parameters which means that the second-order DIARWs can concentrate more energy than the first-order DIARWs, and this outcome agrees with the results of Refs. [20-22]. 

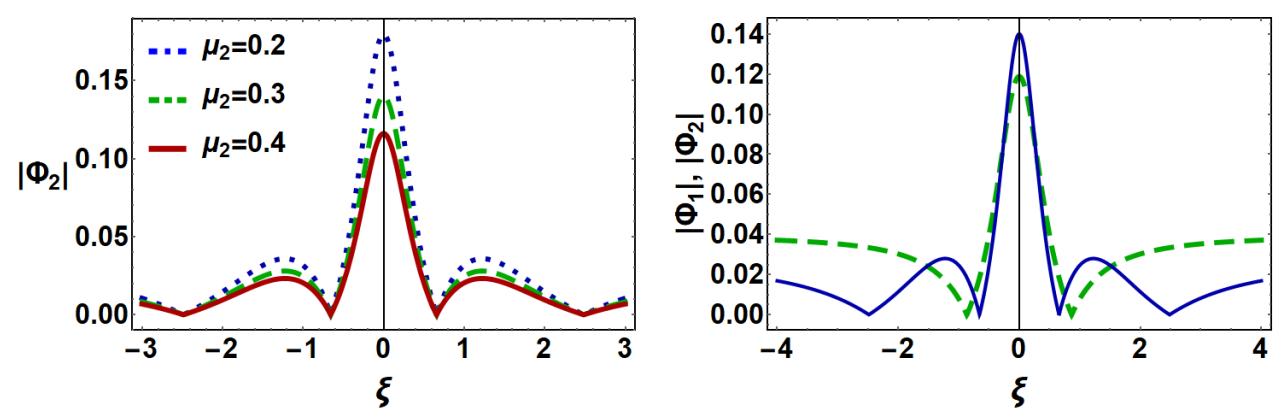

Figure 4. The variation of $\left|\Phi_{2}\right|$ with $\xi$ for different values of $\mu_{2}$ (left panel) and the comparison between the first-order (dashed green curve) and second-order (solid blue curve) rational solutions of NLSE (right panel) when other plasma parameters are $\tau=0, k=1.3, \tilde{\Phi}_{0}=0.5, \kappa=1.8, \rho=1 \times 10^{3}$, $\mu=3 \times 10^{-6}, \mu_{3}=0.05$, and $\omega \equiv \omega_{f}$.

\section{Conclusions}

In this paper, we have studied the stability conditions of the DIAWs in a threecomponent dusty plasma by considering the thermal effect of the positive ions. In our present analysis, the moment of inertia for the formation of the DIAWs is provided by the mass of the positively charged warm ions and negatively charged dust grains, and the restoring force is provided by the thermal pressure of the electrons. A standard NLSE is derived by using RPM. The interaction of the nonlinear $(Q)$ and dispersive $(P)$ coefficients of NLSE can easily divide the modulationally stable and unstable parametric regimes, and the unstable parametric regime also allows us to generate highly energetic DIARWs. The results that have been found from our investigation can be summarized as follows:

- Fast and slow DIA modes have been observed.

- Both modulationally stable (i.e., $P / Q<0$ ) and unstable (i.e., $P / Q>0$ ) parametric regimes of the DIAWs can exist for both fast and slow modes.

- The amplitude of the first and second-order DIARWs decreases (increases) with ion (electron) temperature.

It may be noted here that the gravitational effect is very important but beyond the scope of our present work. The outcomes of present investigation can be useful in explaining the DIARWs in cometary tails [1-3], planetary rings [1], Earth's ionospheres [1], magnetosphere [2], ionosphere [2], aerosols in the astrosphere [3-5], nebula, and interstellar medium [4], etc.

Author Contributions: All authors contributed equally to complete this work. All authors have read and agreed to the published version of the manuscript.

Funding: The research received no external funding.

Institutional Review Board Statement: Not applicable.

Informed Consent Statement: Not applicable.

Data Availability Statement: Data sharing not applicable—no new data generated.

Acknowledgments: The authors are grateful to anonymous reviewers for their constructive suggestions which have significantly improved the quality of our manuscript.

Conflicts of Interest: The authors declare no conflict of interest.

\section{References}

1. Eslami, P.; Mottaghizadeh, M.; Pakzad, H.R. Nonplanar dust-ion acoustic solitary waves in warm plasma with superthermal electrons. IEEE Trans. Plasma Sci. 2013, 41, 3589. [CrossRef]

2. Shahmansouri, M.; Tribeche, M. Dust acoustic localized structures in an electron depleted dusty plasma with two-suprathermal ion-temperature. Astrophys. Space Sci. 2012, 342, 87. [CrossRef]

3. Saini, N.S.; Singh, K. Head-on collision of two dust ion acoustic solitary waves in a weakly relativistic multicomponent superthermal plasma. Phys. Plasmas 2016, 23, 103701. [CrossRef] 
4. Shukla, P.K.; Mamun, A.A. Introduction to Dusty Plasma Physics; Institute of Physics: Bristol, UK, 2002.

5. Zaman, D.M.S; Mannan, A.; Chowdhury, N.A.; Mamun, A.A. Dust-acoustic rogue waves in opposite polarity dusty plasma featuring nonextensive statistics. High Temp. 2020, 58, 789. [CrossRef]

6. Boufendi, L.; Bouchoule, A. Industrial developments of scientific insights in dusty plasmas. Plasma Sources Sci. Technol. 2002, 11, 211. [CrossRef]

7. Gill, T.S.; Bains, A.S.; Bedi, C. Modulational instability of dust acoustic solitons in multicomponent plasma with kappa-distributed electrons and ions. Phys. Plasmas 2010, 17, 013701. [CrossRef]

8. Alinejad, H.; Mahdavi, M.; Shahmansouri, M. Modulational instability of ion-acoustic waves in a plasma with two-temperature kappa-distributed electrons.

Astrophys. Space Sci. 2014, 352, 571. [CrossRef]

9. Gharaee, H.; Afghah, S.; Abbasi, H. Modulational instability of ion-acoustic waves in plasmas with superthermal electrons.

Phys. Plasmas 2011, 18, 032116. [CrossRef]

10. Ferdousi, M.; Sultana, S.; Hossen, M.M.; Miah, M.R.; Mamun, A.A. Dust-acoustic shock excitations in $\kappa$-nonthermal electron depleted dusty plasmas. Eur. Phys. J. D 2017, 71, 102. [CrossRef]

11. Rahman, M.; Chowdhury, N.A.; Mannan, A.; Mamun, A.A. Dust-acoustic rogue waves in an electron-positron-ion-dust plasma medium. Galaxies 2021, 9, 31. [CrossRef]

12. Jahan, S.; Haque, M.N., Chowdhury, N.A.; Mannan, A.; Mamun, A.A. Ion-acoustic rogue waves in double pair plasma having non-extensive particles. Universe 2021, 7, 63. [CrossRef]

13. Sikta, J.; Chowdhury, N.A.; Mannan, A.; Sultana, S.; Mamun, A.A. Electrostatic dust-acoustic rogue waves in an electron depleted dusty plasma. Plasma 2021, 4, 230. [CrossRef]

14. Maksimovic, M.; Gary, S.P.; Skoug, R.M. Solar wind electron suprathermal strength and temperature gradients: Ulysses observations. J. Geophys. Res. 2000, 105, 18337. [CrossRef]

15. Pierrard, V.; Lazar, M. Kappa distributions: Theory and applications in space plasmas. Sol. Phys. 2010, 267, 153. [CrossRef]

16. Vasyliunas, V.M. A survey of low-energy electrons in the evening sector of the magnetosphere with OGO 1 and OGO 3. J. Geophys. Res. 1968, 73, 2839. [CrossRef]

17. Amin, M.R.; Morfill, G.E.; Shukla, P.K. Modulational instability of dust-acoustic and dust-ion-acoustic waves. Phys. Rev. E 1998, 58, 6517. [CrossRef]

18. Jukui, X.; He, L. Modulational instability of cylindrical and spherical dust ion-acoustic waves. Phys. Plasmas 2003, 10, 339. [CrossRef]

19. Saini, N.S.; Kourakis, I. Dust-acoustic wave modulation in the presence of superthermal ions. Phys. Plasmas 2008, $15,123701$. [CrossRef]

20. Guo, S.; Mei, L. Three-dimensional dust-ion-acoustic rogue waves in a magnetized dusty pair-ion plasma with nonthermal nonextensive electrons and opposite polarity dust grainsa. Phys. Plasmas 2014, 21, 082303. [CrossRef]

21. Guo, S.; Mei, L.; Sun, A. Nonlinear ion-acoustic structures in a nonextensive electron-positron-ion-dust plasma: Modulational instability and rogue waves. Ann. Phys. 2012, 332, 38. [CrossRef]

22. Guo, S.; Mei, L.; Shi, W. Rogue wave triplets in an ion-beam dusty plasma with superthermal electrons and negative ions. Phys. Lett. A 2013, 377, 2118. [CrossRef]

23. Almutalk, S.A.; El-Tantawy, S.A.; El-Awady, E.I.; El-Labany, S.K. On the numerical solution of nonplanar dust-acoustic super rogue waves in a strongly coupled dusty plasma. Phys. Lett. A 2019, 16, 1937. [CrossRef]

24. Denra, R.; Paul, S.; Ghosh, U.; Sarkar, S. Nonlinear dust-acoustic wave propagation in a Lorentzian dusty plasma in presence of negative ions. J. Plasma Phys. 2018, 84, 5. [CrossRef]

25. Dubinov, A.E.; Kitayev, I.N. Nonlinear periodic backward dust acoustic waves. Planet. Space Sci. 2021, 195, 105142. [CrossRef]

26. El-Labany, S.K.; El-Taibany, W.F.; El-Tantawy, A.A.; Zedan, N.A. Effects of double spectral electron distribution and polarization force on dust acoustic waves in a negative dusty plasma. Contrib. Plasma Phys. 2020, 60, e202000049. [CrossRef]

27. El-Taibany, W.F.; El-Labany, S.K.; Behery, E.E.; Abdelghany, A.M. Nonlinear dust acoustic waves in a self-gravitating and opposite-polarity complex plasma medium. Eur. Phys. J. Plus 2019, 134, 457. [CrossRef]

28. Chahal, B.S.; Singh, M.; Saini, N.S. Dust ion acoustic freak waves in a plasma with two temperature electrons featuring Tsallis distribution. Phys. A 2018, 491, 935. [CrossRef]

29. El-Labany, S.K.; El-Shewy, E.K.; Abd El-Razek, H.N.; El-Rahman, A.A. Dust-Ion Acoustic Freak Wave Propagation in a Nonthermal Mesospheric Dusty Plasma. Plasma Phys. Rep. 2017, 43, 576. [CrossRef]

30. Mandi, L.; Mondal, K.K.; Chatterjee, P. Analytical solitary wave solution of the dust ion acoustic waves for the damped forced modified Korteweg-de Vries equation in q-nonextensive plasmas. Eur. Phys. J. Spec. Top. 2019, 228, 2753. [CrossRef]

31. Paul, A.; Bandyopadhyay, A.; Das, K.P. Dust ion acoustic solitary structures at the acoustic speed in the presence of nonthermal electrons and isothermal positrons. Plasma Phys. Rep. 2019, 45, 466. [CrossRef]

32. Sinha, A.; Sahu, B. dust-ion-acoustic waves in unmagnetized 4-component plasma. Adv. Space Res. 2021, 67, 1244. [CrossRef]

33. Farooq, M.; Ahmad, M. Dust ion acoustic waves in four component magnetized dusty plasma with effect of slow rotation and superthermal electrons. Phys. Plasmas 2017, 24, 123707. [CrossRef]

34. Das R.; Karmakar K. Fast and slow modes on dust ion acoustic solitary waves in a warm plasma. Int. J. Sci. Res. $2013,3,63$.

35. Mowafy A.E. Propagation of the dust ion acoustic waves in inhomogeneous warm dusty plasma. Afr. Rev. Phys. 2012, 7, 0032. 
36. Heera, N.M.; Akter, J.; Tamanna, N.K.; Chowdhury, N.A.; Rajib, T.I.; Sultana, S.; Mamun, A.A. Ion-acoustic shock waves in a magnetized plasma featuring super-thermal distribution. AIP Adv. 2021, 11, 055117. [CrossRef]

37. Banik, S.; Shikha, R.K.; Noman, A.A.; Chowdhury, N.A.; Mannan, A.; Roy, T.S.; Mamun, A.A. First and second-order dust-ionacoustic rogue waves in non-thermal plasma. Eur. Phys. J. D 2021, 75, 43. [CrossRef]

38. Shikha, R.K.; Chowdhury, N.A.; Mannan, A.; Mamun, A.A. Electrostatic dust-acoustic envelope solitons in an electron-depleted plasma. Contrib. Plasma Phys. 2021, 61, e202000117. [CrossRef]

39. Khondaker, S.; Mannan, A.; Chowdhury, N.A.; Mamun, A.A. Rogue waves in multi-pair plasma medium. Contrib. Plasma Phys. 2019, 59, e201800125. [CrossRef]

40. Dubinov, A.E. On a widespread inaccuracy in defining the Mach number of solitons in a plasma. Plasma Phys. Rep. 2009, 35, 991. [CrossRef]

41. Saberian, E.; Esfandyari-Kalejahi, A.; Afsari-Ghazi, M. Nonlinear dust-acoustic structures in space plasmas with superthermal electrons, positrons, and ions. Plasma Phys. Rep. 2017, 43, 83. [CrossRef]

42. Shukla, P.K.; Silin, V. Dust ion-acoustic wave. Phys. Scr. 1992, 45, 508. [CrossRef]

43. Barkan, A.; Angelo, N.D.; Merlino, R.L. Experiments on ion-acoustic waves in dusty plasmas. Planet. Space Sci. 1996, 44, 239. [CrossRef]

44. Kourakis, I.; Shukla, P.K. Modulational instability and localized excitations of dust-ion acoustic waves. Phys. Plasmas 2003, 10, 3459. [CrossRef]

45. Kourakis, I.; Shukla, P.K. Exact theory for localized envelope modulated electrostatic wavepackets in space and dusty plasmas. Nonlinear Proc. Geophys. 2005, 12, 407. [CrossRef]

46. Fedele, R. Envelope solitons versus solitons. Phys. Scr. 2002, 65, 502. [CrossRef]

47. Noman, A.A.; Chowdhury, N.A.; Mannan, A.; Mamun, A.A. Dust-acoustic envelope solitons in super-thermal plasmas. Contrib. Plasma Phys. 2019, 59, e201900023. [CrossRef]

48. Akhmediev, N.; Ankiewicz, A.; Soto-Crespo, J.M. Rogue waves and rational solutions of the nonlinear Schrödinger equation. Phys. Rev. E 2009, 80, 026601. [CrossRef]

49. Ankiewicz, A.; Devine, N.; Akhmediev, N. Are rogue waves robust against perturbations? Phys. Lett. A 2009, 373, 3997. [CrossRef]

50. Bandyopadhyay, P.; Konopka, U.; Khrapak, S.A.; Morfill, G.E.; Sen, A. Effect of polarization force on the propagation of dust acoustic solitary waves. New J. Phys. 2010, 12, 073002. [CrossRef]

51. Zadorozhny, A.M. Effects of charged dust on mesospheric electrical structure. Adv. Space Res. 2001, 28, 1095. [CrossRef]

52. Wang, Y.L.; Guo, X.Y.; Li, Q.S. Nonlinear dust acoustic waves in strongly coupled dusty plasmas with charged dust particles. Commun. Theor. Phys. 2016, 65, 247. [CrossRef]

53. Bandyopadhyay, P.; Prasad, G.; Sen, A.; Kaw, P.K. Experimental study of nonlinear dust acoustic solitary waves in a dusty plasma. Phys. Rev. Lett. 2012, 101, 065006. [CrossRef]

54. Shalini; Saini N.S. Dust ion acoustic rogue waves in superthermal warm ion plasma. J. Plasma Phys. 2015, 81, 905810316. 